\title{
NANOPARTICLE CHARACTERISATION FOR ECOTOXICOLOGICAL STUDIES USING IMAGING AND NON-IMAGING BASED TOOLS
}

\author{
R. TANTRA, D. GOHIL, S. KALIYAPPAN \& S. JING \\ National Physical Laboratory, Teddington, Middlesex, United Kingdom.
}

\begin{abstract}
There is a great deal of concern about the safety of nanoparticles and their effect on the environment; this has led to a recent Organisation for Economic Co-operation and Development (OECD) drive for testing the toxicity of industrially relevant nanomaterials. A fundamental aspect of the research is the need to measure particle size and particle concentration accurately and reliably, as these two parameters will influence toxicity. The current paper presents a study that aims to characterise $\mathrm{CeO}_{2}$ nanoparticles for the purpose of ecotoxicological studies, and this has resulted in a two-part study that is presented in this paper. The aim of the first part of the study was to observe if small nanosized clusters $(<1 \mu \mathrm{m})$ existed when dispersed in four ecotoxicological media (i.e. fish, daphnia, seawater and de-ionised water); this was done qualitatively using Scanning Electron Microscopy. The second part of the study aimed to explore the feasibility of Nanoparticle Tracking Analysis (NTA) as a suitable tool to characterise not only particle size but also particle number concentration. This involved dispersing $\mathrm{CeO}_{2}$ in DI water and diluting the stock solution into nine different concentrations. Particle size and number data were then acquired using NTA; particle size data were compared to the corresponding Dynamic Light Scattering (DLS) response. The results were as follows: (a) although the majority of the nanomaterials were large aggregates (at least few microns in dimensions), smaller clusters $(<800 \mathrm{~nm})$ were shown to be present in all four media; it is the fate of these small size clusters that should be monitored if hypothesis relating toxicity to particle size holds true, (b) as a tool NTA yielded small particle size compared to DLS measurements and the limit of quantification is shown to be $>0.1 \mathrm{mg} / \mathrm{L}$, as NTA tracks individual particles and does not suffer limitations observed with DLS, in which larger particles can potentially mask signal of smaller particles and (c) calibration curve for number concentration by NTA did not yield a linear response; the non-linear response observed should be further investigated if NTA is going to be used to measure particle number concentration, particularly in the low concentration range.
\end{abstract}

Keywords: agglomerates, aggregation, aquatic ecotoxicity, $\mathrm{CeO}$, characterisation, dispersion, DLS, nanoparticles, NTA, SEM.

\section{INTRODUCTION}

There is an ongoing concern that environmental exposure to engineered nanomaterials may result in significant adverse effects [1]. The uncertainty surrounding nanotoxicity has lead standards organisations to drive the research on a global scale. As a result, the Organization for Economic Co-operation and Development (OECD) has launched a sponsorship program on nanoparticle safety assessment on nanomaterial testing at an international level. PROSPEcT is a project that envelopes the development of ecotoxicology test protocols for representative (industrially relevant) nanomaterials in support of the OECD Sponsorship Programme and it is part of the UK's contribution to test cerium oxide $\left(\mathrm{CeO}_{2}\right)$ and zinc oxide $(\mathrm{ZnO})$ (Fig. 1).

As shown in Fig. 1, the main research activities under PROSPEcT are divided into three areas of research: (a) nanoparticle characterisation, (b) ecotoxicological testings and (c) exploration and development of new techniques for nanoparticle characterisation; it is envisaged that the information obtained will be useful for the purpose of safety risk assessment development.

The current state of research on nanoecotoxicity testing can be summarised in a diagram, in Fig. $2 \mathrm{~b}$. 
Nanoparticle
Characterisation
Ecotoxicological

testings

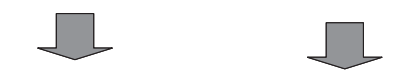

\section{PROSPEcT project. UK's} contribution $\left(\mathrm{CeO}_{2}\right.$ and $\mathrm{ZnO}$ nanomaterials)

OECD

Nanoparticles

Safety Assessment

New techniques for

nanoparticle characterisation

Figure 1: Flow chart illustrating how the UK PROSPEcT project contributes towards the OECD sponsorship program for Nanomaterial Testing.

(a)

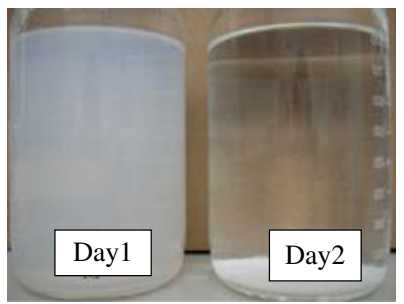

SHOULD WE BE WORRIED?

(b)
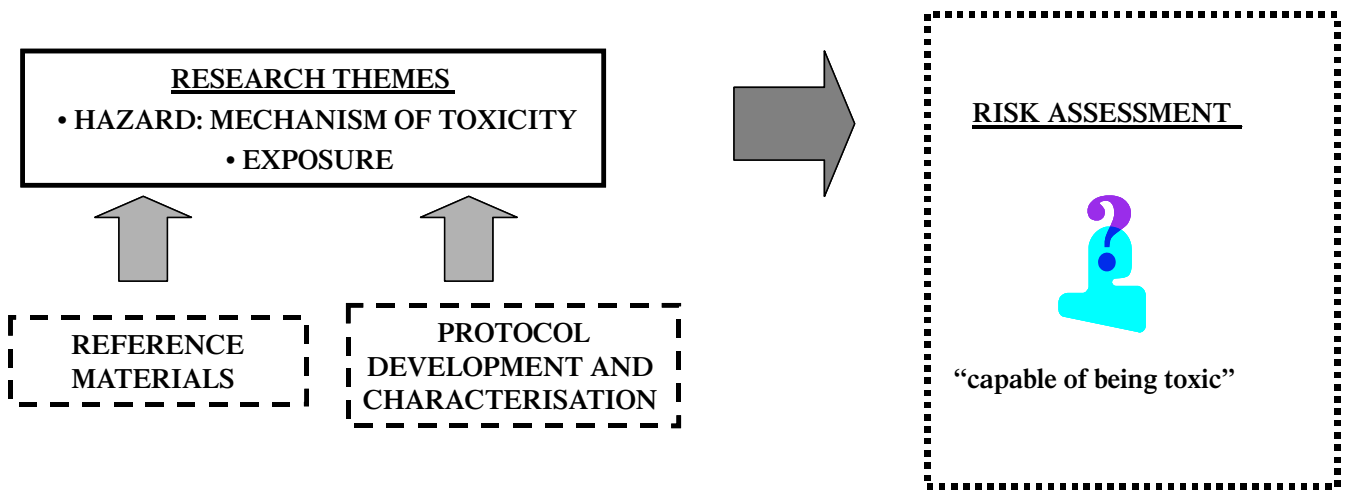

Figure 2: $\mathrm{CeO}_{2}$ in ecotoxicological media. (a) Typical visual sedimentation when nanoparticles are dispersed in an ecotoxicological media (either fish, daphnea or seawater) and (b) current research themes to study toxicity and data input towards risk assessment. 
In a recent study [2], it was observed by conducting simple 'visual sedimentation' experiments (Fig. 2a), that nanomaterials such as $\mathrm{CeO}_{2}$ have a tendency to fall out of solution within a few days; this is very much the case when nanomaterials are suspended in the ecotoxicological relevant media in stagnant or still water conditions.

Hence, it is the degree of interaction between aquatic ionic species and dynamics of aggregate formation that is of key interest.

Currently, nanoecotoxicological research themes (Fig. 2b) fall into two categories: (a) toxicological hazard and (b) exposure risks; data will eventually feed into the development of assessments that help to control risk of nanomaterials in the environment [3]. The current risk assessment guidelines for nanomaterials, of 'capable of being toxic', are unclear. This unclear guideline is mainly due to the inconclusive and sometimes contradictory findings from the hazard and exposure studies conducted by different researchers and this in turn is due to the lack of: (a) suitable reference materials and (b) commonly agreed protocols for testing (both in terms of physicochemical characterisation and bioassay tests). An important factor for the latter is in finding the right tools for nanoparticle characterisation in ecotoxicological studies [3]. Out of the various parameters used for nanoparticle characterisation, (a) particle size and (b) particle concentration are of huge importance. Particle size has often been used to assess toxicological significance by most studies, as researchers are reporting that smaller particles are more toxic than their conventional counterparts [4]. Particle size measurements have often been made using conventional techniques, either based on a population based method (such as Dynamic Light Scattering, DLS) or imaging based techniques (such as Scanning Electron Microscope, SEM) [5]. The use of such routine tools for nanoparticle characterisation for ecotoxicological investigations has been successfully implemented previously [6].

The measurement of particle concentration is of importance specifically for exposure type studies, in particular if we are to understand dose-response relationship. In many cases, the 'dose' of nanoparticles given to a test system is mainly determined by mass. However, researchers have argued this dose metric may not be of nanotoxicological significant and it is the measurement of surface area and number, rather than mass that will be more applicable as dosage metrics for nanoparticles $[7,8]$. Currently, there are only a handful of techniques that can make surface area and number measurements; the Brunauer, Emmett and Teller (BET) measures surface area but is only applicable if the nanomaterials are packed in a solid form [9]. Nonetheless, there are tools that belong to a new generation of analysis tools and this includes Nanoparticle Tracking Analysis (NTA), which is able to directly measure particle number concentration. LIBS (Laser Induced Breakdown Spectroscopy) is another technique that can measure particle number concentration $[10,11]$. However, both techniques are relatively new and their application in nanoecotoxicological investigations is new; thus validation of these techniques are very much needed prior to their use in these studies. NTA has an added advantage over LIBS, in that it is not so specialised and commercially suited for routine measurements. Another advantage of NTA is that it is a noninvasive technique and is able to simultaneously measure particle size. Particle size measurement using the NTA method involves tracking the motion of individual particles using a digital camera and subsequently eporting the hydrodynamic diameter of the particle as calculated through the Stokes-Einstein equation [12].

In this paper, the focus of the study is twofold:

1. To observe (qualitatively) the relative ratios of large aggregates to small nanosize clusters when the nanomaterials are dispersed in the four liquid media.

2. To explore the feasibility of NTA for particle size and particle number measurements and to conduct a comparative study with conventional techniques such as DLS and SEM. 
To assess point (1), dispersions of $\mathrm{CeO}_{2}$ were prepared in four different media (seawater, media of fish, Daphnia and DI water) and SEM was used to observe large aggregates compared to the smaller size aggregates. To evaluate the feasibility of NTA to measure particle number concentration, dispersions of $\mathrm{CeO}_{2}$ were prepared in DI water only and this stock subsequently diluted to nine different concentrations. The corresponding particle number concentrations were measured using NTA and results calibrated against the nine concentrations. Particle size measurements from NTA were also compared to corresponding DLS response.

\section{EXPERIMENTAL}

\subsection{Materials and protocols}

DI water from Millipore, MilliQ system was used to prepare all aqueous solutions and suspensions. Ecotox media was prepared as follows: (a) Seawater $-25 \mathrm{~g}$ per L of Tropic Marine Sea Salt (Tropical and Marine Limited) was prepared. (b) Daphnia freshwater media. Salts ( $196 \mathrm{mg} \mathrm{CaCl} \cdot 2 \mathrm{H}_{2} \mathrm{O}, 82 \mathrm{mg}$ $\mathrm{MgSO}_{4} \cdot 7 \mathrm{H}_{2} \mathrm{O}, 65 \mathrm{mg} \mathrm{NaHCO}, 0.002 \mathrm{mg} \mathrm{Na} \mathrm{SeO}_{3}$ as obtained by appropriate dilutions of a $2 \mathrm{mg} /$ $\mathrm{ml}$ stock solution) were dissolved in $1 \mathrm{~L}$ of DI water. Upon continued stirring, DI water was further added so that the final $\mathrm{pH} \sim 7.5$ and conductivity was between $\sim 360$ and $480 \mu \mathrm{S} / \mathrm{cm}$. End volume $\sim 1$ to 1.5 L. (c) Fish freshwater media. This was prepared in three separate steps. First, salts $(11.76 \mathrm{~g}$ $\mathrm{CaCl}_{2} \cdot 2 \mathrm{H} 2 \mathrm{O}, 4.93 \mathrm{~g} \mathrm{MgSO}_{4} \cdot 7 \mathrm{H}_{2} \mathrm{O}, 2.59 \mathrm{~g} \mathrm{NaHCO}_{3}, 0.23 \mathrm{~g} \mathrm{KCl}$ ) were dissolved separately in $1 \mathrm{~L}$ of DI water to make four separate stock solutions. Second, $25 \mathrm{~mL}$ of each salt stock solution was aliquoted into a clean bottle and diluted in DI water (made up to $1 \mathrm{~L}$ volume). Third, $200 \mathrm{ml}$ of the stock solution from Step 2 was aliquoted and further diluted with DI water (made up to $1 \mathrm{~L}$ volume). For long-term storage, these ecotox solutions were autoclaved and kept refrigerated until needed.

Nanograin $\left(\mathrm{CeO}_{2}\right.$, with an average particle size of $\sim 50$ to $\left.70 \mathrm{~nm}\right)$ was donated by Umicore Belgium. Nanoparticles were dispersed using the protocol as previously reported [2]. Briefly, this involved weighing the nanoparticle powder into small clean vials using an analytical mass balance. Dispersion was carried out by adding the appropriate liquid media (fish, daphnia, seawater or DI water) dropwise and mixing using a spatula so as to produce a thick paste before adding $15 \mathrm{ml}$ of liquid media and stirring gently, using the same spatula. The subsequent de-agglomeration step was carried out using an ultrasonic probe (130 Watt Ultrasonic Processors); this was done by inserting the ultrasonic probe tip (6 $\mathrm{mm} \mathrm{Ti}$ ) half way down the $15 \mathrm{ml}$ volume of dispersed nanoparticles and sonication was carried out with $90 \%$ amplitude for $20 \mathrm{~s}$. After sonication, the nanoparticle suspension was diluted using the appropriate liquid media, in order to make up to $1 \mathrm{~L}$ total volume; a glass rod was used to gently mix the final dispersion, to ensure homogeneity. The dispersions (in the four different media) were stored in separate pre-cleaned $1 \mathrm{~L}$ media bottles and left undisturbed; sedimentation was monitored in the four separate bottles throughout 3 days. After a period of 3 days, each bottle was mixed and aliquot was taken out for analysis under the SEM.

For the purpose of investigating the feasibility of NTA to be used as a tool for nanoparticle size and number measurements, a stock dispersion of $500 \mathrm{mg} / \mathrm{L}$ was prepared and appropriate dilutions with DI water were made from this stock. In order to obtain representative samples when subsampling, the dispersion was agitated sufficiently (using a glass rod) prior to aliquoting the correct amount and diluting further with DI water. The final concentrations were: 500, 200, 100, 50, 10, 1 , $0.1,0.01,0.001 \mathrm{mg} / \mathrm{L}$ and these were used to calibrate the NTA method for particle number concentrations. These samples were also used to measure the quantification limit for particle size measurements using three different techniques: NTA, DLS and SEM. To minimise any variations in the nanoparticle dispersion, all measurements (using the three different tools) were performed simultaneously straight after the sub-sampling step. 


\subsection{Instrumentations}

\subsubsection{Scanning electron microscopy analysis}

SEM images were recorded using a Carl Zeiss Supra 40 field-emission SEM, in which the optimal spatial resolution of the microscope was a few nanometres. In-Lens detector images were acquired at an accelerating voltage of $15 \mathrm{kV}$, working distance of $\sim 3 \mathrm{~mm}$ and a tilt angle $0^{\circ}$. For analysis of the 'as received' nanoparticle powder, a small scoop of the nanoparticle powder was sprinkled over an SEM carbon adhesive disc; one side of the carbon disc was placed securely on a metal stub, whilst the other side was exposed to the nanoparticle powder. Excess powder on top of the disc was removed by gently tapping the stub on its side until an even (light) coating of powder on the surface was apparent. Elemental analysis was performed on the SEM platform using the Energy Dispersive X-ray spectroscopy (EDX) technique, to indicate the purity of the as-received powders.

For analysis of nanoparticles in dispersion, $1 \mathrm{ml}$ of the appropriate liquid dispersion was aliquoted on to a poly-L-lysine coated microscope glass slide (Fisher Scientific, UK). The liquid sample was incubated for a period of $5 \mathrm{~min}$ at room temperature $\left(\sim 20^{\circ} \mathrm{C}\right)$, in which no substantial visible signs of evaporation was observed. After $5 \mathrm{~min}$, the substrate was immersed in DI water, in order to dislodge any loosely bound nanoparticles on the surface. The substrate was then subsequently allowed to air dry in an opened glove box overnight prior to being sputtered with thin film of gold; gold is often as the conductive material in order to prevent charging on the specimen surface. The device used to deposit the gold was an Edwards S150B sputter coater unit; sputtering was conducted under vacuum ( 7 mbar), while passing pure, dry argon into the coating chamber. Typical plate voltage and current were $1200 \mathrm{~V}$ and $15 \mathrm{~mA}$, respectively. The sputtering time was approximately $10 \mathrm{~s}$, which resulted in an estimated gold thickness of not more than $\sim 2 \mathrm{~nm}$ being deposited on top of the substrate. The appropriateness of this sample preparation protocol, for the trapping nanoparticles on to a poly-L-lysine coated substrate, has been previously validated when using NIST-certified $100 \mathrm{~nm}$ latex beads (supplied by Agar Scientific, UK) and results published elsewhere [5].

\subsubsection{DLS (particle size and zeta-potential) analysis}

The DLS instrument employed for particle size analysis measurements was a Zetasizer Nano ZS (Malvern Instruments, UK) with $633 \mathrm{~nm}$ red laser. Particle size was obtained from measuring the time dependent fluctuation of scattered light arising from the suspension of the nanoparticles undergoing random Brownian motion. Analysis was carried out using a disposable folded capillary cell (DTS1060). For the analysis, the nanoparticle sample of the desired concentration was flushed through a folded capillary cell and the measurement was carried out on the second filling; a sufficient sample volume was used to completely cover the electrodes of the cell. To avoid air bubbles in the cell, the sample was injected slowly and analysis was only carried out if there were no visible air bubble inclusions present. After successful inspection, the cell was placed into the Zetasizer and equilibrated at $20^{\circ} \mathrm{C}$ (close to the average temperature in the laboratory) for 2 min prior to the particle size measurements; the measurement temperature was set and maintained by the Peltier elements in the sample holder of the instrument.

\subsubsection{Nanoparticle Tracking Analysis}

Nanoparticle Tracking Analysis (NTA) from Nanosight, UK (LM10 model) was used to measure the particle size and number concentration. This system used a $638 \mathrm{~nm}$ wavelength laser, which was focused through a $500 \mu \mathrm{l}$ of sample; for all experiments a $20 \times$ objective was used to collect the light. 
Samples were injected into an optical unit using sterile syringes and nanoparticles visible within the field of view were separately tracked on a frame-by-frame basis, by a CCD camera (variable electronic shutter duration ranged from $20 \mu \mathrm{s}$ to $30 \mathrm{~ms}$ ). The resulting output is thus a collection of images (of resolution $640 \times 480$ pixels) captured for a movie length $(60 \mathrm{~s}$ duration), typically from 1000 to 5000 frames (with a frame rate of 60 frames/s). This technique was calibrated by measurement of NIST traceable latex beads (nominal $\sim 100 \mathrm{~nm}$, Duke Scientific). The analysis was repeated three times to ensure that measurement results are repeatable. The software (NTA 2.0 Software programme) was used to acquire, record and determine the particle size and number count information.

\subsection{Data analysis}

Data collected from all instruments (apart from SEM) were imported to Excel and the appropriate mean and standard deviations from the set of (three) replicates calculated. Results are plotted as mean values with error bars of one standard deviation.

\section{RESULTS AND DISCUSSION}

\subsection{Nanoparticle dispersion}

Figure $3 \mathrm{a}$ shows the SEM image of the as-received powder of $\mathrm{CeO}_{2}$ nanomaterial. It is clear that the powder showed evidence of extensive aggregation and agglomeration (fusion of particles). This was expected for nanoparticles due to the presence of strong interparticle forces that promote higher levels of aggregation and subsequently agglomeration [13]. The purity of the nanomaterial is shown in the inset, using the EDX technique. Results showed that there were no peaks that corresponded to any impurity elements and suggested that the nanomaterial was of high purity; the carbon peak emanated from the carbon stub that was used to fix the powder for SEM examination. No doubt that a platform such as SEM-EDX is considered to extremely powerful as potentially it can offer complementary chemical information in addition to structural information. This is important as it is able to provide positive identification of the nanomaterial under study and thus is useful for the detection of contamination that can arise from a variety of different sources, e.g. most likely during the handling of the nanoparticles. Although EDX has the potential to yield elemental maps, it has to be remembered that this spectroscopic based techniques is sensitive to heavier elements such as $\mathrm{P}, \mathrm{S}, \mathrm{K}$ and $\mathrm{Ca}$ [14]. Figure $3 \mathrm{~b}$ shows the DLS particle size distribution when the nanomaterial (at $50 \mathrm{mg} / \mathrm{L}$ ) was dispersed using the protocol specified in the Method section. The results indicated that a bi-modal distribution was present, with the first distribution having a mean particle hydrodynamic diameter of $\sim 200 \mathrm{~nm}$, with a polydispersity index (PDI) of 0.2 (this suggests a reasonably narrow size distribution). PDI is a parameter that the width of the distribution; Zetasizer DLS reports a PDI value between 0 and 1 , in which a PDI value of 0.1 will represent monodispersity. Values $<0.1$ indicate polydispersity in the sample, with values between 0.1 and 0.25 indicating a narrow particle size distribution [15]. The particle distribution data also suggested that particle sizes greater than a few microns were also present. There is a great deal of uncertainty as to reliability of this actual size in the micron region. DLS is determined by random Brownian motion and so if particles are in the micron size then their movement may no longer be random, with some of the larger ones sedimenting [16]. Overall, the ultrasonic probe used in this study was shown to provide significant energy to successfully break up the aggregates observed in the as-received powders. If compared to other de-agglomerating tools such as an overhead stirrer or homogeniser, it has been shown that the ultrasonic probe is most effective at breaking down aggregates [2]. 
(a)

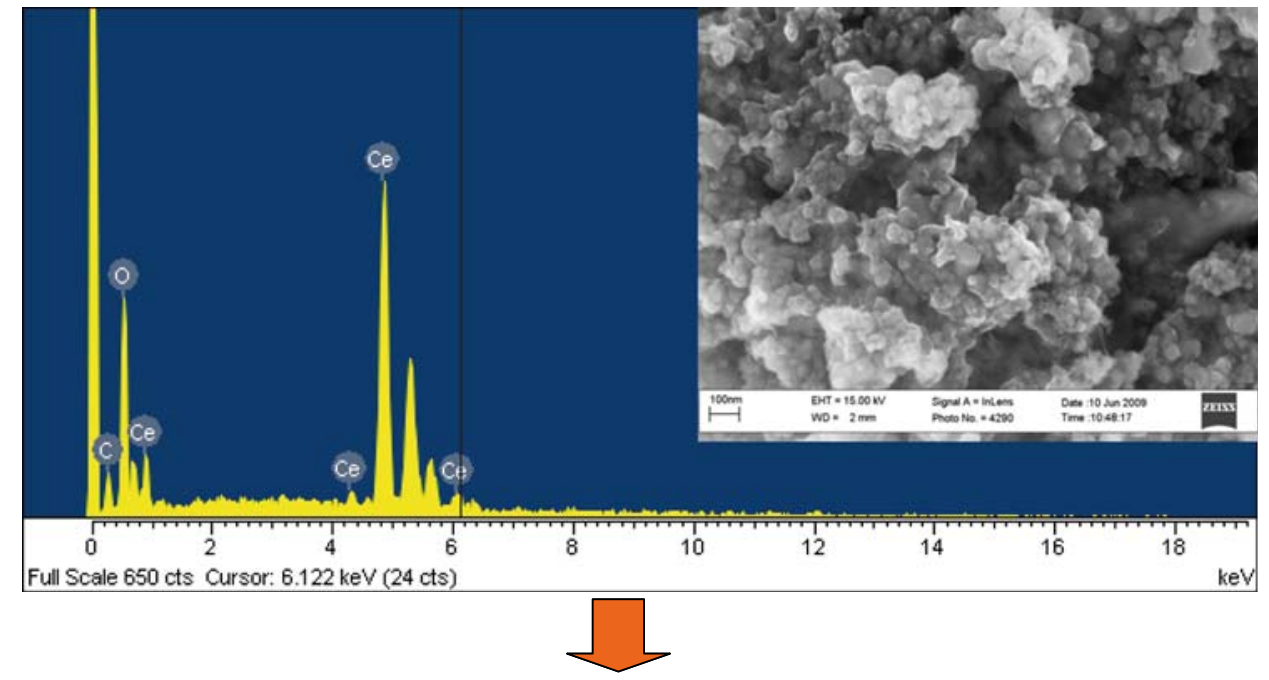

(b)

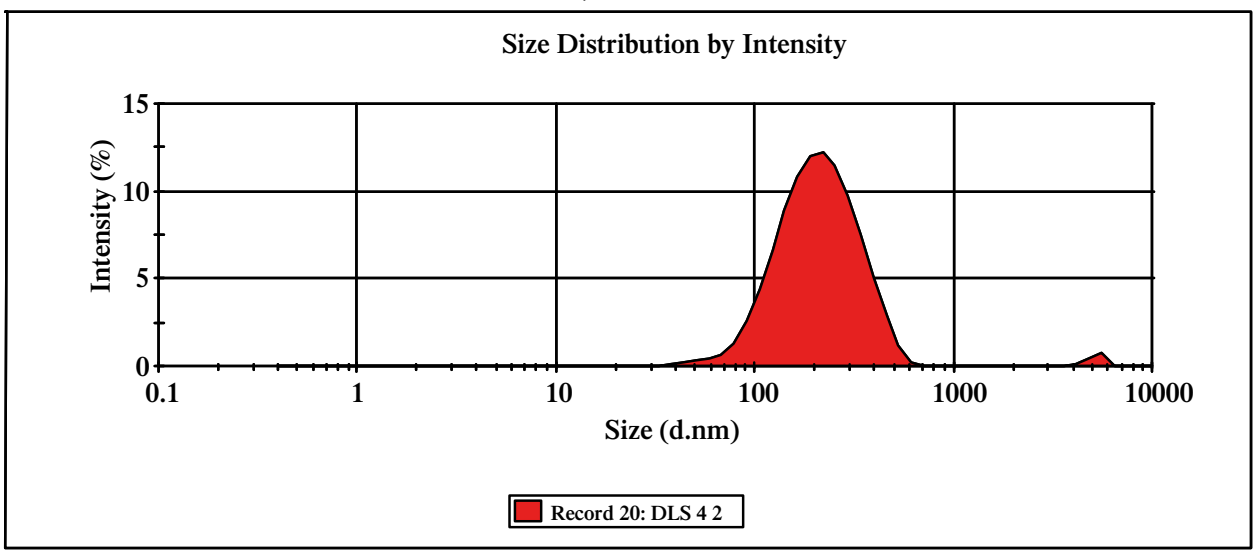

Figure 3: $\mathrm{CeO}_{2}$ : (a) SEM-EDX of the 'as received', (b) DLS particle size distribution (by intensity) after appropriate dispersion $(50 \mathrm{mg} / \mathrm{L}$ in DI water).

\subsection{Visual sedimentation}

Figure 4 shows typical SEM images of the collected nanomaterial after day 3 of the visual sedimentation experiment. In all four media, large clusters of micron size aggregates were clearly evident, as is indicative that these particles will eventually sediment out of the solution.

Eventual particle aggregation is expected in:

1. seawater media, is due to charge screening effects to de-stabilise the system, as offered at these high salt concentrations. This is explained by the classical DVLO (Derjaguin and Landau, Verwey and Overbeek) theory. This theory predicts that stability of a colloidal dispersion is predicted from the particle pair interaction energy (expressed as the sum of an attractive van der Waals term and a repulsive double layer component). If salt concentration is low, then the suspension will be stabilised by an electrostatic energy barrier. Upon increasing salt 
(a)

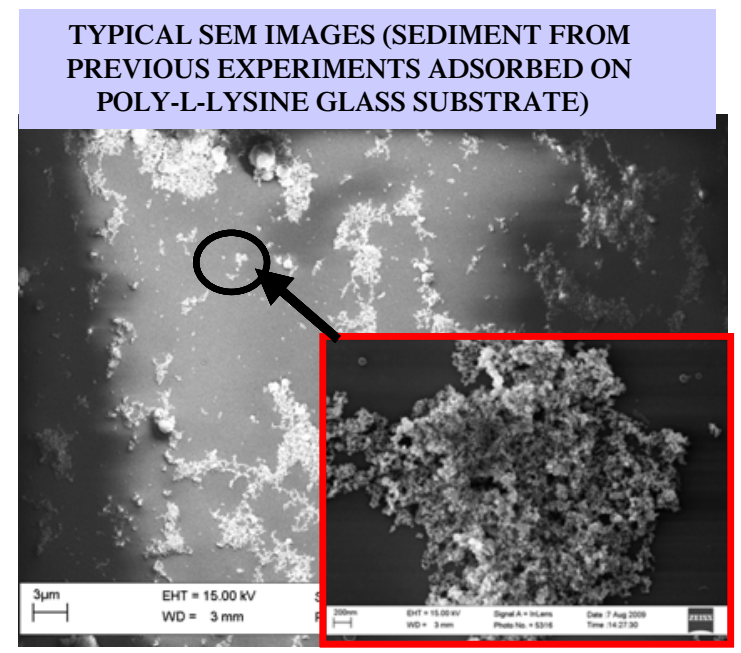

(b)
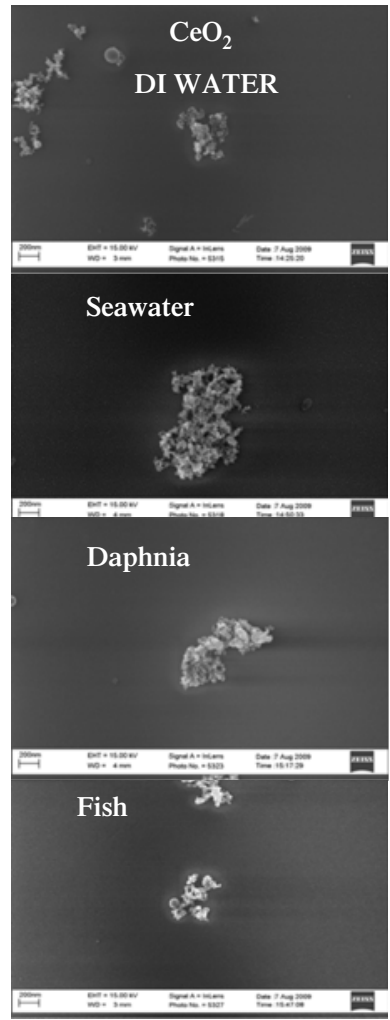

Figure 4: Typical SEM image of nanoparticles collected on poly-L-lysine glass slides, showing the presence of: (a) large micron size aggregates, as found in all four media and (b) smaller nanomaterial aggregates in four media (DI water, Seawater, Daphnia and Fish).

concentration, the electrostatic repulsive forces are progressively screened and thereby the height of the barrier is decreased [17], resulting in particle collision.

2. DI water, as there is no additive to electrostatically stabilise the nanoparticles [18].

3. both Daphnea and fish water media consisted of salt concentrations in the few $\mathrm{mM}$ region; the salt concentration was not considered to be sufficiently high enough to cause charge screening effects as observed in seawater and so would not cause de-stabilisation of the particles. With these media, the degree of stabilisation would be governed by factors such as: (a) $\mathrm{pH}$ and (b) the presence of adsorbing ionic species on to the surface of the nanomaterials. Both parameters would significantly affect surface charge and subsequent stability of the nanoparticles dispersed in the solution [18].

Although most of the particles consisted of extremely large aggregates after day 3 , results also showed the presence of some smaller clusters in all four media (Fig. 4). Clusters of particles $<800 \mathrm{~nm}$ in size were clearly present; size here is taken as the spherical equivalent. Results indicated that not all of the particles had flocculated and sedimented out; some particles are left behind as the larger aggregates sediment out. It is likely that the smaller clusters remain in suspension, whilst most of the particles are sedimenting out of the solution. As expected the concentration of 
these smaller clusters were much lower than the larger aggregate counterparts, it is possible that they remain as stable entities in solution as there is inherently greater interparticle distance that will reduce particle collision [19]. If the hypothesis that relates particle size with toxicity holds true (i.e. the smaller the size the higher the toxicological activity), then it is the presence of these smaller size clusters that should be of utmost concern to the aquatic environment and should be monitored in terms of its accumulation and potential toxic impact on the ecosystem.

\subsection{Particle size measurements as measured by various techniques: DLS, NTA and SEM}

Figure 5 shows the mean particle size of the $\mathrm{CeO}_{2}$ dispersion (at $50 \mathrm{mg} / \mathrm{L}$ ) in DI water, at various particle concentrations, as measured by DLS and NTA.

Identifying the limit of quantification for an individual analytical procedure is important, so as to understand when data become unreliable. This is particularly of importance to nanoecotoxicological investigations as researchers in this field are often interested in making measurements under extremely dilute conditions, in the order of less than few ng/L [20]. The DLS plot here shows a quantification limit of $0.1 \mathrm{mg} / \mathrm{L}$ with particle size that ranges between 180 and $220 \mathrm{~nm}$. Upon further dilution, the data value shifts to a much larger value (420 and $650 \mathrm{~nm}$ for 0.01 and $0.001 \mathrm{mg} / \mathrm{L}$, respectively) and these are thought to be erroneous results and such observations have been previously attributed mainly to the inherent homodyne configuration of the DLS optics [21]. NTA on the other hand does not show this highly erroneous result at the extreme dilute particle concentration range. NTA shows a particle size range of $105-156 \mathrm{~nm}$ up to concentration of $0.01 \mathrm{mg} / \mathrm{L}$ (indicating quantification limit of the method at this concentration); further dilution resulted in particle size of $171 \mathrm{~nm}$. Hence, the NTA technique is not as sensitive to erroneous contributions at

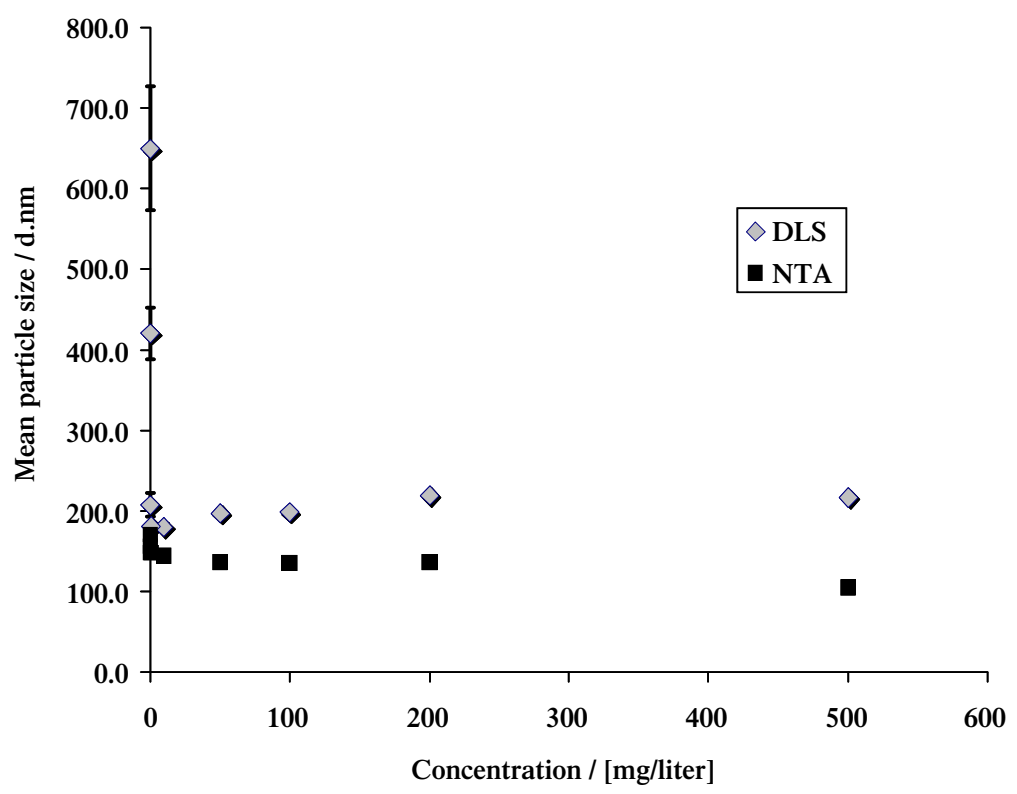

Figure 5: $\mathrm{CeO}_{2}$ dispersed in DI water and the effect of nanoparticle dilution on mean particle size as measured by: (a) Dynamic Light Scattering (DLS) and (b) Nanoparticle Tracking Analysis (NTA). 
extremely low particle concentration; the reason being in the different way that particle is being measured by the different techniques. It is interesting to also note that overall particle size acquired under NTA is smaller than the reported value from the corresponding DLS measurements. This is expected, as the NTA approach does not give the average particle size and thus will not be biased towards the larger particles (as with the DLS). In DLS, larger particles within a polydisperse sample will scatter preferentially in the forward direction and thus will be more represented and mask the smaller particles in suspension [12]. In addition, NTA cannot track large aggregates reliably and so they will not be picked up by the technique; it has been reported by Malloy and Carr that the NTA method is not suitable for analysis above $600 \mathrm{~nm}$ [22]. As observed from the SEM images (Fig. 4) micron size aggregates were indeed present when the nanomaterials are dispersed in an ecotox relevant media and in this sense both techniques would be limited when measuring the larger aggregates.

Figure 6 shows a series of SEM images $\mathrm{CeO}_{2}$ (dispersed in DI water and subsequently adsorbed on the surface of poly-L-lysine substrates), upon changing nanoparticle concentration within the dispersion. It is apparent that the particle size distribution changes dramatically when nanoparticle concentration in the dispersion is diluted from 500 to $10 \mathrm{mg} / \mathrm{L}$.

A much-reduced number of nanoparticles and a tendency for smaller particles adhering to the surface were observed under the extreme dilution conditions. For SEM, the limit of quantification is governed by the adsorption kinetics of the nanoparticles on to the substrates during the sample preparation step. At low nanoparticle concentrations, it is the diffusion rate of the particle that will dominate and this in turn explains why the smaller particles are preferentially adsorbed within a
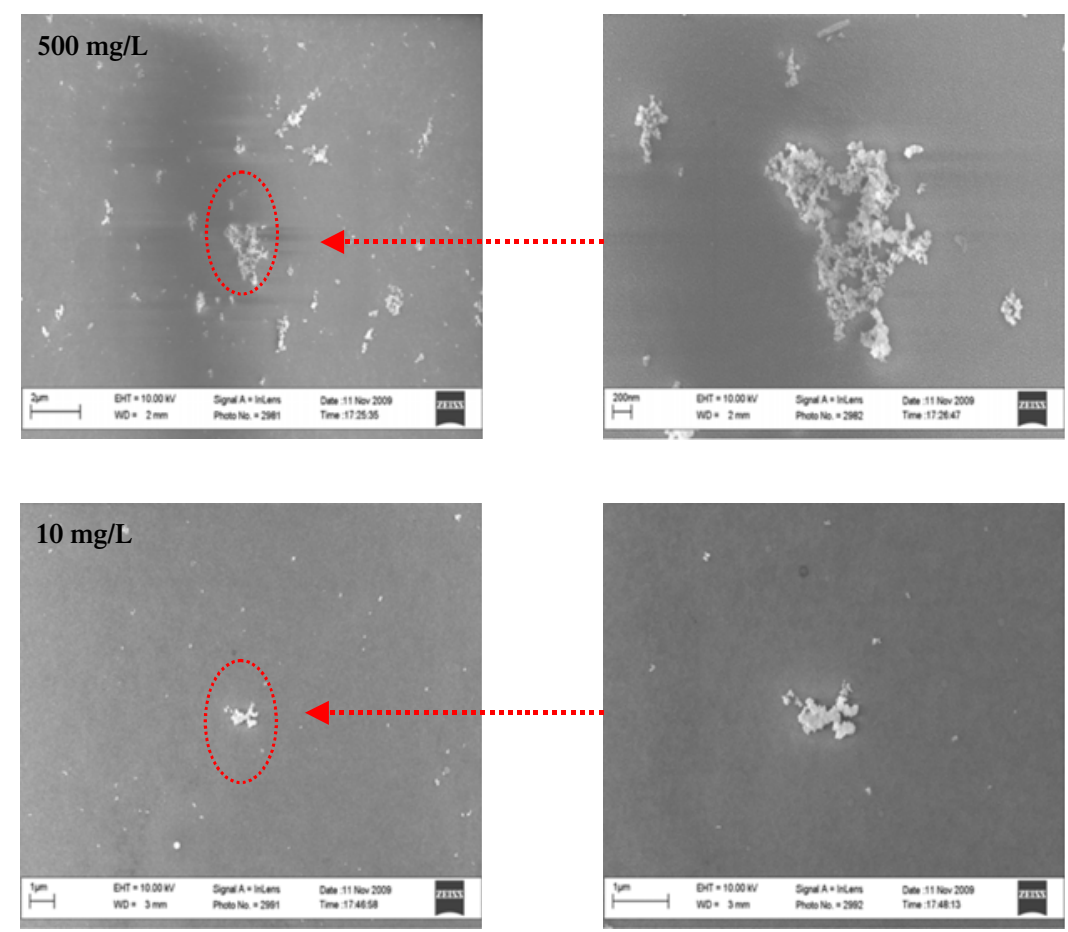

Figure 6: $\mathrm{CeO}_{2}$ dispersed in DI water and the effect of nanoparticle dilution on SEM. This shows that limit of quantification is limited by particle adhesion on the poly-L-lysine substrate. 


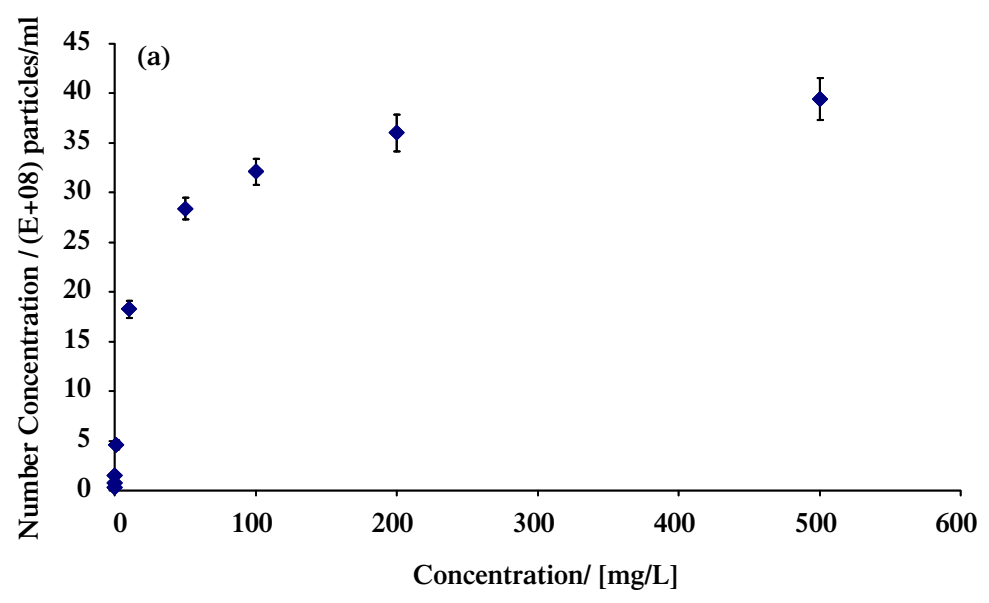

(b)

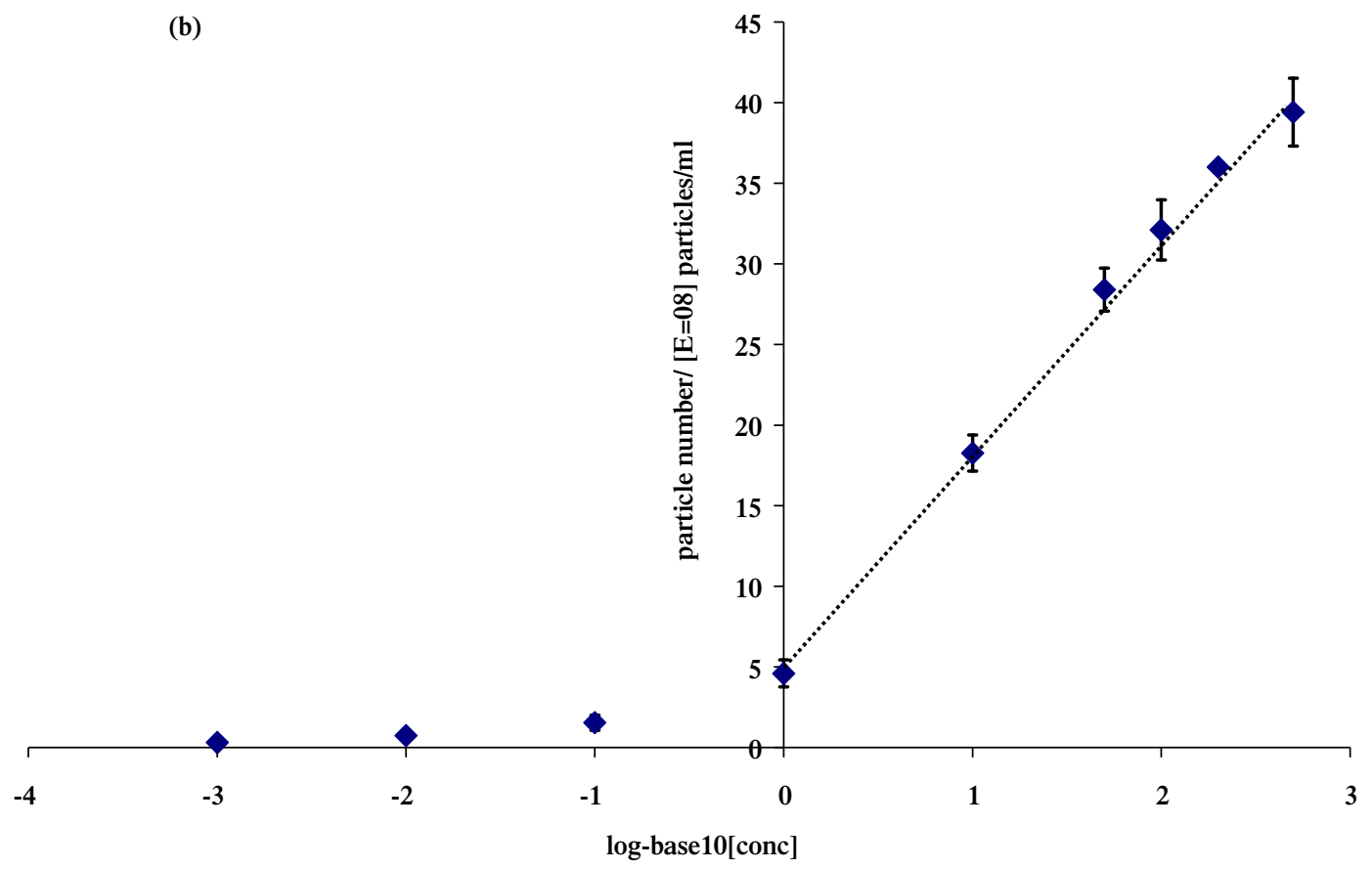

Figure 7: $\mathrm{CeO}_{2}$ dispersed in DI water and the effect of nanoparticle dilution on particle number concentration as measured using Nanoparticle Tracking Analysis number concentration with: (a) concentration by mass (in $\mathrm{mg} / \mathrm{L}$ ) and (b) corresponding log mass concentration. Error bars represent \pm 1 standard deviation.

given period of time [18]. In theory, it is possible to prolong the adsorption period to improve the limit of quantification if using the SEM method, as it may be possible to trap more particles on the surface given sufficiently long adsorption time. However, to do this the protocol should involve immersing the slides into the dispersion, so as to minimise the volume change 
and subsequent evaporation, which will result in particle aggregation occurring during the sample preparation step.

\subsection{Feasibility of NTA for particle number measurements}

Figure 7 a shows the particle number $\mathrm{CeO}_{2}$ concentration calibrated against various (nine) concentrations. The calibration plot shows that going from lower to higher concentration, the NTA response is increasing with concentration until it reaches a point in which the signal starts to saturate after $100 \mathrm{mg} / \mathrm{L}$ concentration. The corresponding log (concentration) response is also nonlinear (as shown in Fig. 7b) across the entire range.

As the sample used for this calibration experiment was made by ensuring careful sub-sampling of the original stock, it was expected that the various particle concentrations (defined here by mass) should thus be proportional to the corresponding number concentration. Hence, the nonlinear response of the calibration plot is slightly unexpected. However, it may be that linearity of response is within a much smaller range than expected. According to a previous study, in which particle number concentration was calibrated upon dilutions for (Duke Scientific) reference standard of monodispersed particles (for both 400 and $200 \mathrm{~nm}$ particle sizes (concentration of $1 \mathrm{wt} \%$ )) over a dilution range of a factor of 10 , it was observed that larger errors were evident at the greater dilutions factors [23].

As a technique the significant advantage of NTA over DLS is in the fact that particle number concentration is directly obtainable, as the number concentration is preferred in nanotoxicological investigation rather than mass concentration [24]. However, the non-linear response of the calibration plot in our study suggests that we would need to understand the source behind this non-linearity, if we are to use the NTA method in order to reliably estimate the particle number concentration.

\section{CONCLUSION}

While most nanoparticles were shown to aggregate out of solution (into micron size particles) when immersed in an ecotoxicological relevant media, some smaller clusters were still present. If this was to occur in a real environmental setting, then there is potential for aquatic organisms to ingest such small particles and through time, these small particles can accumulate and it is the accumulation of dose that can subsequently create a serious ecological problem. A huge challenge in nanotoxicological research is in finding the right tools that can measure particle size and (number or surface area) concentration at extremely dilute particle concentrations, in order to determine mechanism and dose of toxicity. However, it is crucial that measurements are reliable, so that appropriate risk assessment of nanomaterials can be further developed. In this study, we explored the use of NTA to measure particle size and number concentrations of $\mathrm{CeO}_{2}$ dispersions. It was found that particle size from NTA measurement is smaller than that of the corresponding DLS; the difference in size was attributed to the ability for NTA to track individual particle rather than from the collection of an overall population volume signal (as with DLS measurements). Like DLS, the main limitation in the measurement of particle size is if micron size particles are present in the dispersion. In DLS, large particle size can mask the signal from smaller particles, whereas in NTA the technique is not able to reliably track (and estimate size information) of large particles. The main advantage of NTA over DLS, however, is that it can measure particle number concentration simultaneously. Our attempt to calibrate the particle number concentration showed that this response is parabolic in nature and there is a need to understand the source of this non-linearity. 


\section{ACKNOWLEDGEMENTS}

This work was conducted as part of PROSPEcT, which is a public-private partnership between DEFRA, EPSRC and TSB and the Nanotechnology Industries Association (NIA Ltd.) and its members, and was administered by the DEFRA LINK Programme. The authors would like to thank Mr. Jordan Tompkins for initial handling and distribution of the nanoparticles; Neil Harrison and Alex Shard for helpful comments and encouragement. The author is an employee of the Crown and as such this paper is subject to Crown Copyright.

\section{REFERENCES}

[1] Maynard, A.D. \& Pui, D.Y.H., Nanotechnology and occupational health: new technologies - new challenges. Journal of Nanoparticle Research, 9(1), pp. 1-3, 2007. doi:10.1007/s11051-006-9164-8

[2] Tantra, R., Jing, S. \& Gohil, D., Technical issues surrounding the preparation, characterisation and testing of nanoparticles for ecotoxicological studies. Environmental Toxicology 3, ed. V.B. Popov, C.A, WIT Press, pp. 165-176, 2010.

[3] Tiede, K. et al., Detection and characterization of engineered nanoparticles in food and the environment. Food Additives and Contaminants, 25(7), pp. 795-821, 2008. doi:10.1080/02652030802007553

[4] Lundqvist, M., Stigler, J., Elia, G., Lynch, I., Cedervall, T. \& Dawson, K.A., Nanoparticle size and surface properties determine the protein corona with possible implications for biological impacts. Proceedings of the National Academy of Sciences of the United States of America, 105(38), pp. 14265-14270, 2008. doi:10.1073/pnas.0805135105

[5] Tantra, R., Tompkins, J. \& Quincey, P., Characterisation of the de-agglomeration effects of bovine serum albumin on nanoparticles in aqueous suspension. Colloids and Surfaces B: Biointerfaces 75, pp. 275-281, 2010. doi:10.1016/j.colsurfb.2009.08.049

[6] Handy, R.D. et al., The ecotoxicology and chemistry of manufactured nanoparticles. Ecotoxicology, 17(4), pp. 287-314, 2008. doi:10.1007/s10646-008-0199-8

[7] Karakoti, A.S., Hench, L.L. \& Seal, S., The potential toxicity of nanomaterials - the role of surfaces. Jom, 58(7), pp. 77-82, 2006. doi:10.1007/s11837-006-0147-0

[8] Oberdörster, G. et al., Principles for characterizing the potential human health effects from exposure to nanomaterials: elements of a screening strategy. Particle and Fibre Toxicology, 2, p. 8, 2005. doi:10.1186/1743-8977-2-8

[9] Lowell, S., Shields, J.E., Thomas, M.A. \& Thommes, M., Characterization of Porous Solids and Powders: Surface Area, Pore Size and Density (Particle Technology Series), Springer, 1 st edn. 2004. Corr. 2nd printing edition, 2006.

[10] Zheng, Z.K. et al., Research on laser induced breakdown spectroscopy for detection of trace $\mathrm{Cu}$ in polluted soil. Spectroscopy and Spectral Analysis, 29(12), pp. 3383-3387, 2009.

[11] Jung, E.C. et al., Nanoparticle sizing by a laser-induced breakdown detection using an optical probe beam deflection. Applied Physics B-Lasers and Optics, 97(4), pp. 867-875, 2009. doi:10.1007/s00340-009-3780-9

[12] Filipe, V., Hawe, A. \& Jiskoot, W., Critical evaluation of nanoparticle tracking analysis (NTA) by nanosight for the measurement of nanoparticles and protein aggregates. Pharmaceutical Research, 27(5), pp. 796-810, 2010. doi:10.1007/s11095-010-0073-2

[13] Hosokawa, M. et al., Nanoparticle Technology Handbook, Elsevier: Amsterdam, 2007.

[14] Leapman, R.D. \& Hunt, J.A., Comparison of detection limits for Eels and Edxs. Microscopy Microanalysis Microstructures, 2(2-3), pp. 231-244, 1991. doi:10.1051/mmm:0199100202-3023100

[15] Malvern Instruments Ltd., Technical information provided for the Zeta-sizer Nano. 
[16] Berne, B.J. \& Pecora, R., Dynamic Light Scattering: with Applications to Chemistry, Biology, and Physics, Unabridged edition, Dover Publications, 2000.

[17] Hubbard, A.T., Encyclopedia of Surface and Colloid Science, New York Marcel Dekker: New York, 2002.

[18] Kissa, E.E., Dispersions: Characterization, Testing, and Measurement, Marcel Dekker, New York, 1999.

[19] Hiemenz, P.C. \& Rajagopalan, R., Principles of Colloid and Surface Chemistry (Hardcover), Marcel Dekker, New York, 1997.

[20] Simonet, B.M. \& Valcarcel, M., Monitoring nanoparticles in the environment. Anal Bioanal. Chem., 393, pp. 17-21, 2009. doi:10.1007/s00216-008-2484-Z

[21] Tantra, R., Schulze, P. \& Quincey, P., The effect of nanoparticle concentration on zeta-potential meausurement results and reproducibility. Particuology, 8, pp. 279-285, 2010. doi:10.1016/ j.partic.2010.01.003

[22] Malloy, A. \& Carr, B., Nanoparticle tracking analysis - the Halo (TM) system. Particle \& Particle Systems Characterization, 23(2), pp. 197-204, 2006. doi:10.1002/ppsc.200601031

[23] Nanosight, Technical Note M500B How to make Concentration Measurements using NanoSight LM Series Instruments, 2009.

[24] Oberdorster, G., Oberdorster, E. \& Oberdorster, J., Concepts of nanoparticle dose metric and response metric. Environmental Health Perspectives, 115(6), pp. A290, 2007. doi:10.1289/ehp.115-a290a 